UNDERGROUND MINING ENGINEERING 32 (2018) 1-14 $\quad$ UDK 62

\title{
INVESTIGATION OF QUANTITATIVE INDICATORS OF ANISOTROPY BASED ON LONGITUDINAL ELASTIC WAVES PROPAGATION VELOCITIES WITH UNCERTAINTY
}

\author{
Miloš Gligorić1, Jelena Majstorović1, Suzana Lutovac ${ }^{1}$
}

Received: May 11, 2018

Accepted: June 15, 2018

\begin{abstract}
Almost all of rock masses are more or less anisotropic medium. Foliation, layering, cracking and cleavage are some of the rock attributes that indicate to anisotropy presence. Due to the existence of these discontinuities, elastic waves propagation velocities along certain directions are extremely different.

In this paper, we investigated total of 50 samples which are divided into two groups of samples, isotropic and anisotropic samples. Longitudinal elastic waves propagation velocities are measured in the three normal directions and three orthogonal planes, respectively. The main scope of this study is related to interval numbers in order to express uncertainties of the longitudinal elastic waves propagation velocities. Based on this, we have determined quantitative indicators of anisotropy. Obtained results are represented in graphic form.
\end{abstract}

Keywords: anisotropy investigation; longitudinal elastic waves propagation velocities; quantitative indicators of anisotropy; interval numbers

\section{INTRODUCTION}

Anisotropy is the property of rock sample showing different physical characteristics along different directions. Rock masses anisotropy is especially manifested on their following properties: mechanical resistance, elasticity, deformability, water permeability and heat conductivity. Rocks often exhibit a macroscopic anisotropy of mechanical properties (Petružálek et al., 2008). Ultrasonic laboratory experiments on cores show evidence for both layering and fracture related anisotropy in different rock types (Melaku, 2007). Ultrasonic velocities are related to the properties of the stone and they can thus be applied to assess the quality of the stones, to detect any presence of microscopic fissuring and cracks, to evaluate the material decay and to assess the effectiveness of consolidation products (Esbert, 2007; Favaro et al., 2006; Fort et al., 2013; Jasiehko and Stawiski, 2001; Kahraman, 2007; K1lıc, 2006; Myrin and Malaga, 2006; Sousa et al., 2005; Valdelon et al., 1996; Vasanelli et al., 2015; Yavuz et al., 2006).

Measuring method of ultrasonic waves velocity propagation is based on the modelling of the wave propagation process. The process of induction and wave emission is repeated

\footnotetext{
${ }^{1}$ University of Belgrade - Faculty of Mining and Geology

Emails: milos.gligoric@ @rgf.bg.ac.rs; jelena.majstorovic@ @rgf.bg.ac.rs; $\underline{\text { suzana.lutovac@ @rgf.bg.ac.rs }}$
} 
several times per second because of its shortness, so this method is called impulse method. Elastic waves in rock or sample are representing deformation propagation, so depending of type of deformations, waves of different types can be caused:

a) longitudinal waves

b) transversal waves

Today, ultrasonic waves with frequency over $20000 \mathrm{~Hz}$ are applied in elastic properties investigations of different materials, both in laboratories and ,in situ“ conditions. At the end of the seventies in the last century for rock samples investigation (borehole core, blocks, etc.) as for field investigations, Mining department of the Faculty of Mining and Geology purchased an instrument of type PUNDIT (Portable Ultrasonic Non-destructive Digital Indicating Taster) (Gligorić et. al., 2017). This instrument is projected for application in mines (portable), small dimensions, weight only $3 \mathrm{~kg}$ with battery. This device can work on the basis of internal combustion, external combustion and alternating current. It consists of the following basic parts:

- impulse generator

- delay detection device

- receiver

- impulse oscillator

- recording device

Using this instrument, only longitudinal elastic waves propagation velocities can be measured. Based on these elastic waves, we have analyzed the properties of isotropic and anisotropic samples. Anisotropy can be expressed quantitatively by comparing certain characteristics such as physical, mechanical or structural along different directions. In our study, we have measured longitudinal elastic waves propagation velocities in order to determine presence of anisotropy on the two types of rock samples. The investigations implied calculation of quantitative indicators of anisotropy. In order to decrease uncertainty related to external infuencing on devices, we have applied interval numbers. On that way, we upgraded previous paper (Gligorić et. al., 2017) by converting crisp values of the longitudinal elastic waves propagation velocities into interval values. Also, we have created a concept of anisotropy calculation which involves a determination of maximum, minimum and mean value of the longitudinal elastic waves propagation velocities. To calculate quantitative indicators of anisotropy, we have used two approaches to calculate the degree of anisotropy and one for calculating the anisotropy coefficient.

The remainder of the paper is constructed as follows. Section 2 describes basics of interval numbers such as arithmetic operations, maximum and minimum between two interval numbers, ranking of interval numbers and converting interval values into a crisp values. Input parameters which are referred on defining longitudinal elastic waves propagation velocities are given in Section 3. Based on this input parameters, concept of 
calculation of quantitative indicators of anisotropy is presented in Section 4. Section 5 illustrates obtained results and discussions in relation to our investigations represented at Figures 1-6. Also, brief description of statistical analysis is shown in this section. Finally, Section 6 explains the conclusion of our study.

\section{BASICS OF INTERVAL NUMBERS}

The devices for measuring ultrasonic waves are constantly exposed to external influences such as adverse weather conditions, inaccessible field and inexperience of workers. Due to these problems, sometimes the results of measurements are not so accurate and precise. Presence of vague and imprecise data is the basic indicator of uncertainty. In order to overcome these problems it is necessary to apply interval numbers. Interval numbers provide a possibility that the obtained results can be found in some defined boundaries. On that way, measurement errors are reduced to minimum. As it mentioned earlier, crisp values of the longitudinal elastic waves propagation velocities are converted into interval values. These values are commonly defined by suitable interval numbers due to their arithmetic operations simplicity.

The following section gives a brief description of interval numbers, basic properties and operations.

Let $\hat{P}=\left[p^{l}, p^{u}\right]$ and $\hat{Q}=\left[q^{l}, q^{u}\right]$ be a two real closed intervals (Alefeld and Mayer, 2000; Moore et al., 2009; Trindade et al., 2010). The arithmetic operations of addition, subtraction, multiplication and division, for real numbers, are representing using symbol $\circ \in\{+,-, \cdot, \div\}$

Then we define the corresponding operations for intervals $\hat{P}$ and $\hat{Q}$ by $\hat{P} \circ \hat{Q}=$ $\{p \circ q \mid p \in \hat{P}, q \in \hat{Q}\}$

Note: it is necessary to assume that $0 \notin \hat{Q}$ for the operation of division

Interval operations:

1. Addition

$$
\widehat{P}+\hat{Q}=\left[p^{l}+q^{l}, p^{u}+q^{u}\right]
$$

2. Subtraction

$$
\hat{P}-\hat{Q}=\left[p^{l}-q^{u}, p^{u}-q^{l}\right]
$$


3. Multiplication

$$
\hat{P} \cdot \hat{Q}=\left[p^{l} \cdot q^{l}, p^{u} \cdot q^{u}\right]
$$

4. Division

$$
\frac{\hat{P}}{\hat{Q}}=\left[\frac{p^{l}}{q^{u}}, \frac{p^{u}}{q^{l}}\right] \quad q^{l}, q^{u} \neq 0
$$

5. Inversion

$$
\hat{P}^{-1}=\frac{1}{\hat{P}}=\left[\frac{1}{p^{l}}, \frac{1}{p^{u}}\right] \quad p^{l}, p^{u} \neq 0 \quad \hat{Q}^{-1}=\frac{1}{\widehat{Q}}=\left[\frac{1}{q^{l}}, \frac{1}{q^{u}}\right] \quad q^{l}, q^{u} \neq 0
$$

6. Equality

$$
\hat{P}=\hat{Q} \quad \text { if } \quad p^{l}=q^{l} \text { and } p^{u}=q^{u}
$$

7. Degenerated interval

$$
\hat{P}=\left[p^{l}, p^{u}\right] \quad \text { if } \quad p^{l}=p^{u}=p \quad \text { then } \hat{P}=[p, p] \text { called degenerated interval }
$$

8. Mean interval value

Suppose $\hat{S}=\left[s_{i}^{l}, s_{i}^{u}\right], i=1,2, \ldots, N$ is a set of $N$ interval numbers. The mean interval value of $\hat{S}$ is defined as:

$$
E(\hat{S})=\left[\frac{1}{N} \sum_{i=1}^{N} s_{i}^{l}, \frac{1}{N} \sum_{i=1}^{N} s_{i}^{u}\right]
$$

9. Maximum and minimum between two interval numbers

To determine maximum and minimum between two or more interval numbers, we used the Moore Distance. (Trindade et al., 2010). Firstly, we must define a degenerated interval which represent a reference value called zero interval. In relation to zero interval we compute distance to the current interval. That distance shows a range of closeness between zero interval and current interval given by:

$$
D_{M}(\hat{P}, \hat{Z})=\max \left\{\left|p^{l}-z^{l}\right|,\left|p^{u}-z^{u}\right|\right\}
$$


Zero interval: $\hat{Z}=\left[z^{l}, z^{u}\right]=[0,0]$

Ranking of interval numbers, based on Moore Distance, is defined as:

$$
\begin{aligned}
& \text { If } D_{M}(\hat{P}, \hat{Z})>D_{M}(\hat{Q}, \hat{Z}) \text { then } \hat{P}=\max , \hat{Q}=\min \\
& \text { If } D_{M}(\hat{P}, \hat{Z})<D_{M}(\hat{Q}, \hat{Z}) \text { then } \hat{Q}=\max , \hat{P}=\min
\end{aligned}
$$

10. Converting interval number into a crisp value

$$
P=\left[\frac{p^{l}+p^{u}}{2}\right]
$$

\section{THE CONCEPT OF ANISOTROPY CALCULATION}

The total of directions are equal to total number of the orthogonal planes $n=3$ which are mutually normal to each rock sample. Longitudinal elastic waves propagation velocities are measured into three mutually normal directions which are shown as 1 $1^{\prime}, 2-2^{\prime}, 3-3^{\prime}$.

In this study, the total number of samples varies depending on the type of samples. There are two types of samples: isotropic and anisotropic samples. The total number of isotropic samples are 24 and the total number of anisotropic samples are 26. Input parameters are defined as follows:

Let

$\widehat{V I}_{i, \max }=\max \left\{\widehat{V}_{i, 1}, \ldots, \widehat{V}_{i, n}\right\} ; \forall i \in[1,24] ; n=1,2,3$ be a set that describing the maximum of the longitudinal elastic waves propagation velocities for each special isotropic sample including all three directions

$\widehat{V I}_{i, \min }=\min \left\{\widehat{V}_{i, 1}, \ldots, \widehat{V}_{i, n}\right\} ; \forall i \in[1,24] ; n=1,2,3$ be a set that describing the minimum of the longitudinal elastic waves propagation velocities for each special isotropic sample including all three directions

$\widehat{V I}_{j, \max }=\max \left\{\widehat{V}_{j, 1}, \ldots, \widehat{V}_{j, m}\right\} ; \forall j \in[1,2,3] ; m=1,2, \ldots, 24$ be a set that describing the maximum of longitudinal elastic waves propagation velocities for each special direction including all isotropic samples

$\widehat{V I}_{j, \min }=\min \left\{\widehat{V}_{j, 1}, \ldots, \widehat{V}_{j, m}\right\} ; \forall j \in[1,2,3] ; m=1,2, \ldots, 24$ be a set that describing the minimum of longitudinal elastic waves propagation velocities for each special direction including all isotropic samples 
$\widehat{V I}_{i, \text { mean }}=\operatorname{mean}\left\{\widehat{V}_{i, 1}, \ldots, \widehat{V}_{i, n}\right\} ; \forall i \in[1,24] ; n=1,2,3$ be a set that describing the average of the longitudinal elastic waves propagation velocities for each special isotropic sample including all three directions

$\widehat{V I}_{j, \text { mean }}=\operatorname{mean}\left\{\widehat{V}_{j, 1}, \ldots, \widehat{V}_{j, m}\right\} ; \forall j \in[1,2,3] ; m=1,2, \ldots, 24$ be a set that describing the average of the longitudinal elastic waves propagation velocities for each special direction including all isotropic samples

$\widehat{V A}_{i, \max }=\max \left\{\widehat{V}_{i, 1}, \ldots, \widehat{V}_{i, n}\right\} ; \forall i \in[1,26] ; n=1,2,3$ be a set that describing the maximum of the longitudinal elastic waves propagation velocities for each special anisotropic sample including all three directions

$\widehat{V A}_{i, \min }=\min \left\{\hat{V}_{i, 1}, \ldots, \widehat{V}_{i, n}\right\} ; \forall i \in[1,26] ; n=1,2,3$ be a set that describing the minimum of the longitudinal elastic waves propagation velocities for each special anisotropic sample including all three directions

$\widehat{V A}_{j, \max }=\max \left\{\widehat{V}_{j, 1}, \ldots, \widehat{V}_{j, m}\right\} ; \forall j \in[1,2,3] ; m=1,2, \ldots, 26$ be a set that describing the maximum of longitudinal elastic waves propagation velocities for each special direction including all anisotropic samples

$\widehat{V A}_{j, \min }=\min \left\{\widehat{V}_{j, 1}, \ldots, \widehat{V}_{j, m}\right\} ; \forall j \in[1,2,3] ; m=1,2, \ldots, 26$ be a set that describing the minimum of longitudinal elastic waves propagation velocities for each special direction including all anisotropic samples

$\widehat{V A}_{i, \text { mean }}=\operatorname{mean}\left\{\widehat{V}_{i, 1}, \ldots, \widehat{V}_{i, n}\right\} ; \forall i \in[1,24] ; n=1,2,3$ be a set that describing the average of the longitudinal elastic waves propagation velocities for each special anisotropic sample including all three directions

$\widehat{V A}_{j, \text { mean }}=\operatorname{mean}\left\{\widehat{V}_{j, 1}, \ldots, \widehat{V}_{j, m}\right\} ; \forall j \in[1,2,3] ; m=1,2, \ldots, 24$ be a set that describing the average of the longitudinal elastic waves propagation velocities for each special direction including all anisotropic samples

\section{QUANTITATIVE INDICATORS OF ANISOTROPY}

As it mentioned in the previous paper (Gligorić et. al., 2017), two types of rock samples, isotropic and anisotropic, are investigated. Longitudinal elastic waves propagation velocities are measured for both of samples. Obtained results can be used to estimate quantitative indicators of anisotropy: anisotropy coefficient $K$ and degree of anisotropy $D$. The following section provides a detailed description of the concept of calculation and formulas.

There are several ways to determine the degree of anisotropy and anisotropy coefficient. Our investigation of the degree of anisotropy is based on longitudinal elastic waves 
propagation velocities. Elastic waves propagation velocities through isotropic and anisotropic samples are different which has been proven using formulas for calculation. The calculation of degree of anisotropy is divided into two parts. First part is referred to determination degree of anisotropy for each special sample. The second part shows determination degree of anisotropy for each special direction. The next part of calculation process describes investigation of anisotropy coefficient for each special sample and each special direction, respectively. It should be emphasized that the whole calculation process is covered applying interval numbers.

Degree of anisotropy for isotropic and anisotropic samples is calculated with following equations (Melaku, 2007).

The first one approach is related to each special isotropic samples and defined as follows:

$$
\widehat{D I}_{i, \text { max }}=\frac{\widehat{V I}_{i, \text { max }}-\widehat{V I}_{i, \text { min }}}{2 \cdot \widehat{V I}_{i, \text { max }}} \cdot 100 \%
$$

The second one approach for each special isotropic samples is defined as following equation:

$$
\widehat{D I}_{i, \text { min }}=\frac{\widehat{V I}_{i, \max }-\widehat{V I}_{i, \text { min }}}{2 \cdot \widehat{V I}_{i, \text { min }}} \cdot 100 \%
$$

Degree of anisotropy for each special anisotropic sample using two previous approaches can be expressed as following equations:

$$
\begin{aligned}
\widehat{D A}_{i, \text { max }} & =\frac{\widehat{V A}_{i, \text { max }}-\widehat{V A}_{i, \text { min }}}{2 \cdot \widehat{V A}_{i, \text { max }}} \cdot 100 \% \\
\widehat{D A}_{i, \text { min }} & =\frac{\widehat{V A}_{i, \text { max }}-\widehat{V A}_{i, \text { min }}}{2 \cdot \widehat{V A}_{i, \text { min }}} \cdot 100 \%
\end{aligned}
$$

Degree of anisotropy for isotropic and anisotropic sample is calculated for each special direction as follows:

$$
\begin{aligned}
\widehat{D I}_{j, \text { max }} & =\frac{\widehat{V I}_{j, \text { max }}-\widehat{V I}_{j, \text { min }}}{2 \cdot \widehat{V I}_{j, \text { max }}} \cdot 100 \% \\
\widehat{D I}_{j, \text { min }} & =\frac{\widehat{V I}_{j, \text { max }}-\widehat{V I}_{j, \text { min }}}{2 \cdot \widehat{V I}_{j, \text { min }}} \cdot 100 \%
\end{aligned}
$$




$$
\begin{aligned}
\widehat{D A}_{j, \text { max }} & =\frac{\widehat{V A}_{j, \text { max }}-\widehat{V A}_{j, \text { min }}}{2 \cdot \widehat{V A}_{j, \text { max }}} \cdot 100 \% \\
\widehat{D A}_{j, \text { min }} & =\frac{\widehat{V A}_{j, \text { max }}-\widehat{V A}_{j, \text { min }}}{2 \cdot \widehat{V A}_{j, \text { min }}} \cdot 100 \%
\end{aligned}
$$

Anisotropy coefficient for each special isotropic and anisotropic sample and for each special direction can be calculated with following equations (Melaku, 2007):

$$
\begin{aligned}
\widehat{K I}_{i, \text { mean }} & =\frac{\widehat{V I}_{i, \text { max }}-\widehat{V I}_{i, \text { min }}}{\widehat{V I}_{i, \text { mean }}} \cdot 100 \% \\
\widehat{K I}_{j, \text { mean }} & =\frac{\widehat{V I}_{j, \text { max }}-\widehat{V I}_{j, \text { min }}}{\widehat{V I}_{j, \text { mean }}} \cdot 100 \% \\
\widehat{K A}_{i, \text { mean }} & =\frac{\widehat{V A}_{i, \text { max }}-\widehat{V A}_{i, \text { min }}}{\widehat{V A}_{i, \text { mean }}} \cdot 100 \% \\
\widehat{K A}_{j, \text { mean }} & =\frac{\widehat{V A}_{j, \text { max }}-\widehat{V A}_{j, \text { min }}}{\widehat{V A}_{j, \text { mean }}} \cdot 100 \%
\end{aligned}
$$

\section{RESULTS AND DISCUSSION}

Using arithmetic operation (3), obtained results are represented as a converted interval values into a crisp values for a both of approaches of the calculation the degree of anisotropy and also for anisotropy coefficient.

Degree of anisotropy for each special isotropic and anisotropic sample and for each special direction, respectively, is calculated by Equations (4), (5), (6), (7), (8), (9), (10) and (11) based on arithmetic operations (2) and (3). Anisotropy coefficient for each special isotropic and anisotropic sample and for each special direction, respectively, is calculated by Equations (12), (13), (14) and (15) based on arithmetic operations (1), (2) and (3).

Figure 1 and Figure 2 are referred on the calculation of the degree of anisotropy for each special isotropic and anisotropic samples including all of the directions. It is clearly visible that degrees of anisotropy for isotropic samples are approximately same and the differences are very small using both of approaches. On the other side, degrees of anisotropy for anisotropic samples are different and deviations are quite high. 


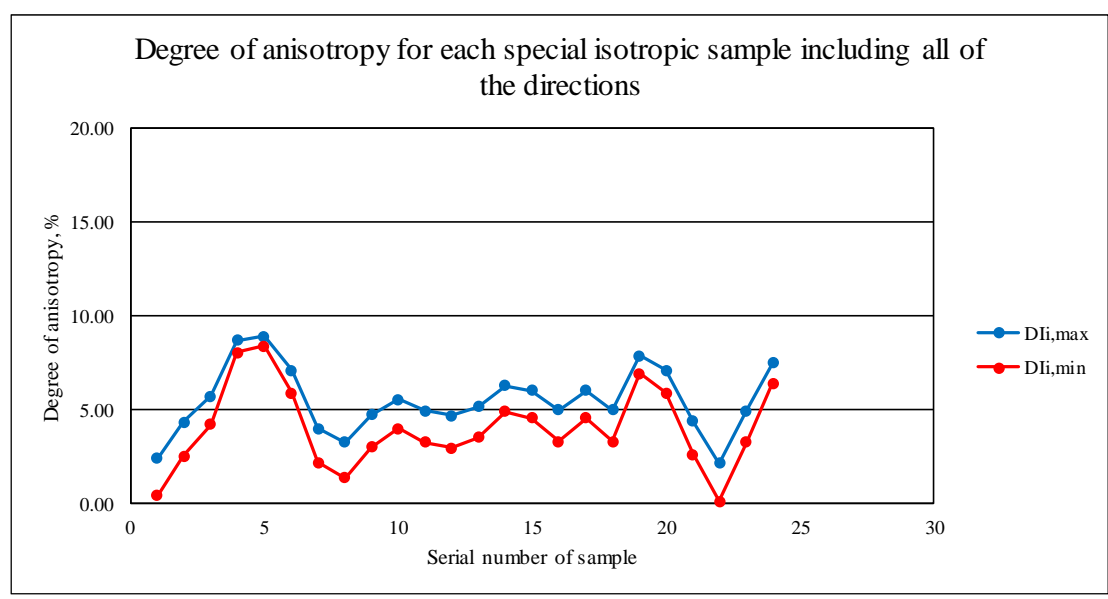

Figure 1 Degrees of anisotropy for each special isotropic sample including all of the directions

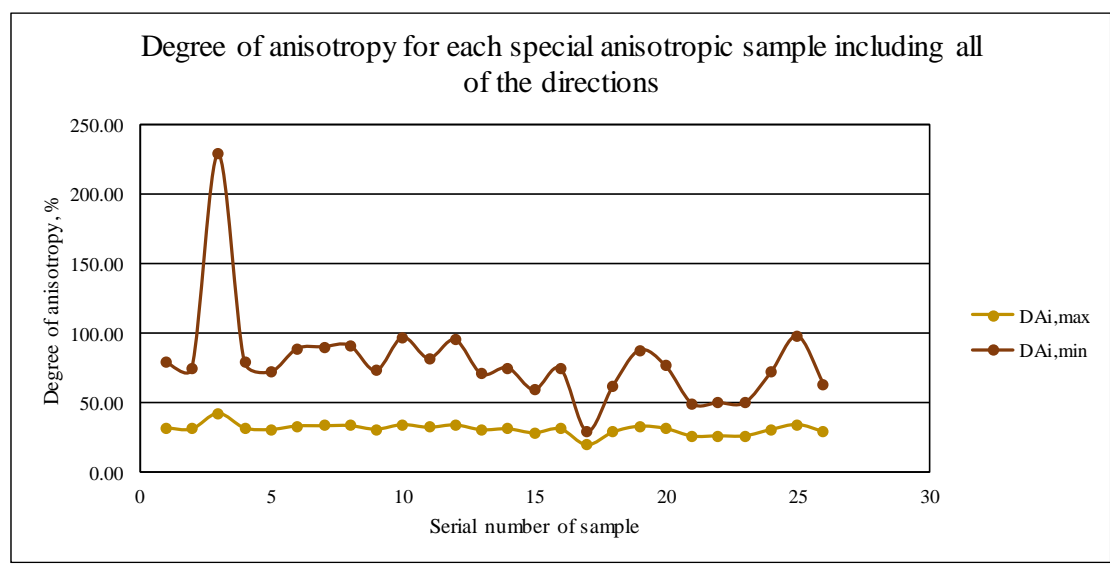

Figure 2 Degrees of anisotropy for each special anisotropic sample including all of the directions

Figure 3 shows anisotropy coefficients for each special isotropic and anisotropic sample including all of the directions. Differences between isotropic and anisotropic samples are obvious. Anisotropy coefficients for isotropic samples cover a less range of values than the anisotropy coefficients for anisotropic samples. It means that anisotropy coefficients for isotropic samples follow each other and values tends to keep a straight line. In contrast, anisotropy coefficients for anisotropic samples have no tend to be a straight line and differences between each other are high and clearly expressed. 


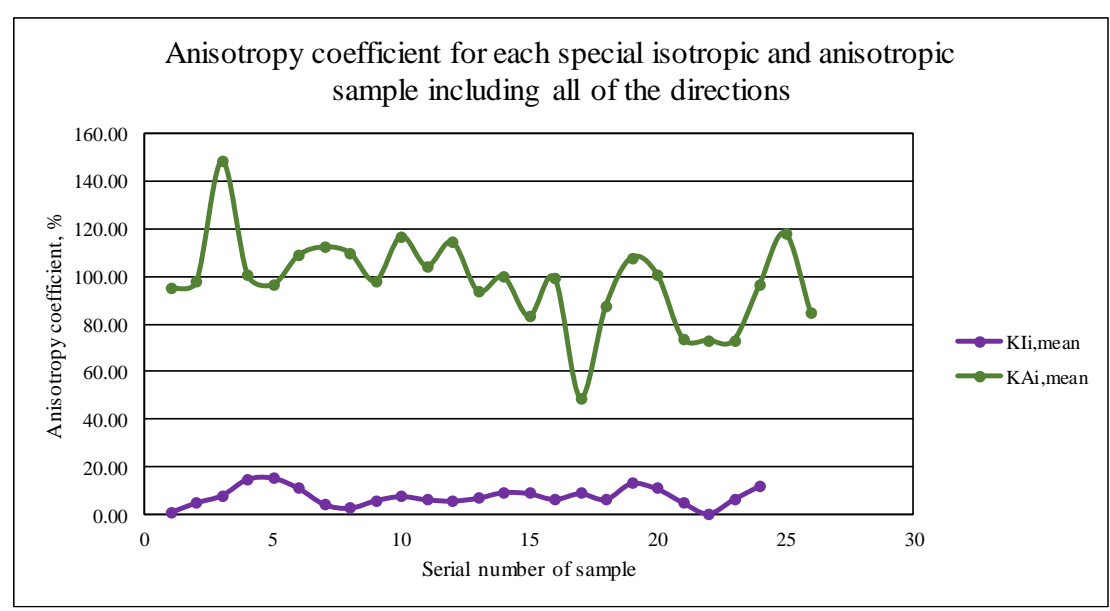

Figure 3 Anisotropy coefficients for each special isotropic and anisotropic sample including all of the directions

Figure 4 and Figure 5 represent the degree of anisotropy for each special direction for all isotropic and anisotropic samples. Degrees of anisotropy for each special direction show similar values in all three directions using both of approaches. Thus, obtained results indicate that a balance has been established for all three directions which further implies that it is an isotropic sample. As regards of anisotropic samples, degrees of anisotropy for each special direction have no similar values. Differences are very high and there is no uniformity between both of approaches from which we can conclude that it is an anisotropic sample.

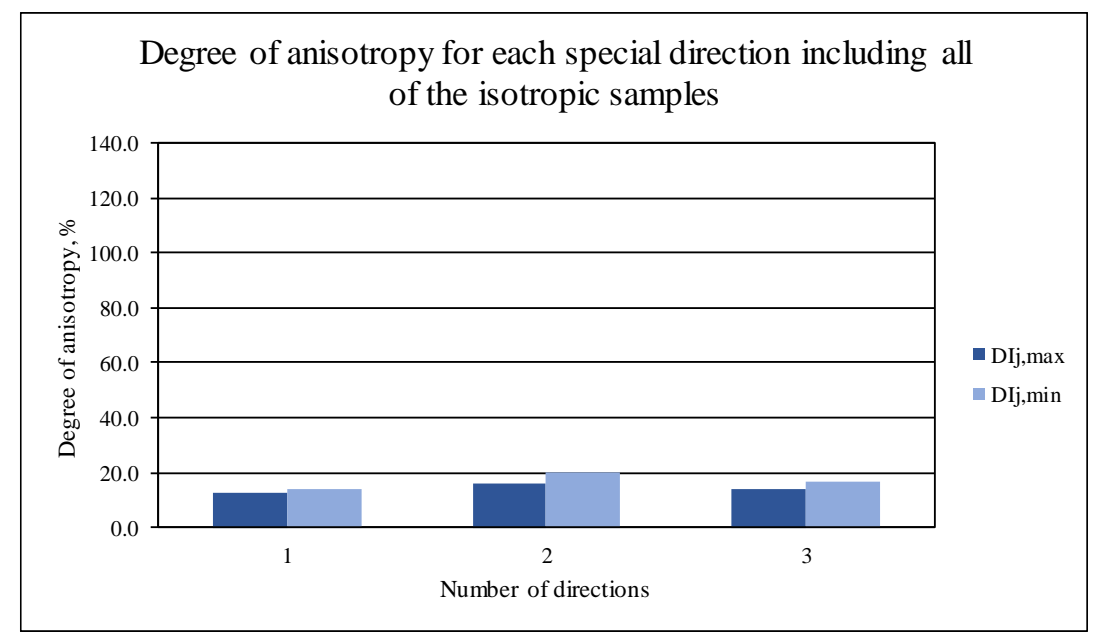

Figure 4 Degrees of anisotropy for each special direction including all of the isotropic samples 


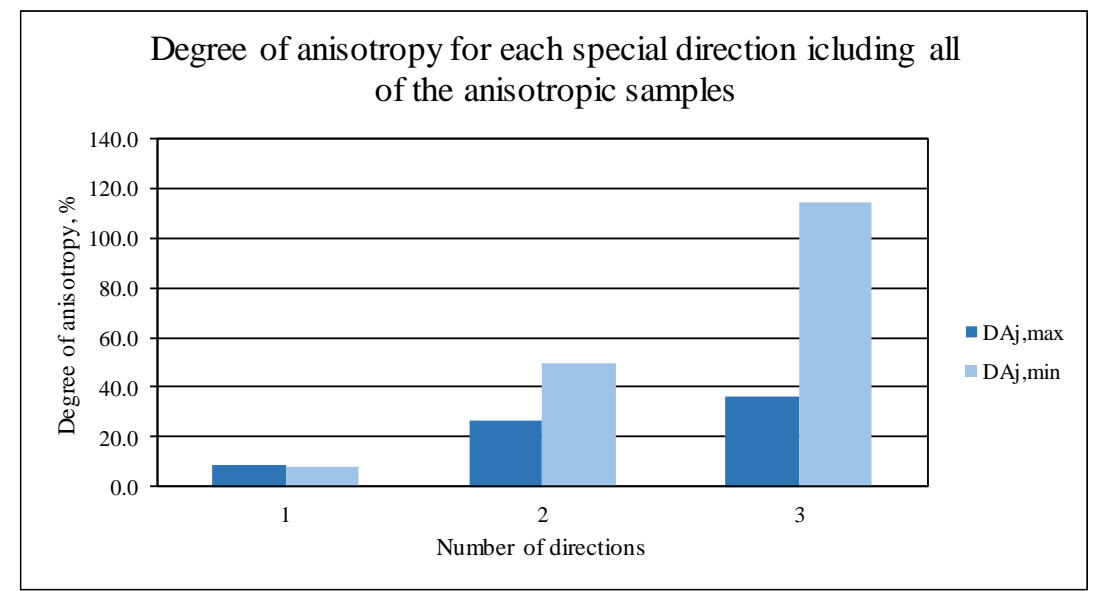

Figure 5 Degrees of anisotropy for each special direction including all of the anisotropic samples

Figure 6 gives a comparative review of anisotropy coefficients for each special direction including all of the isotropic and anisotropic samples. As explained in the earlier segments, this Figure also shows obvious difference between isotropic and anisotropic samples. In this case, we can easily notice an incompatibility between anisotropy coefficients of isotropic and anisotropic samples.

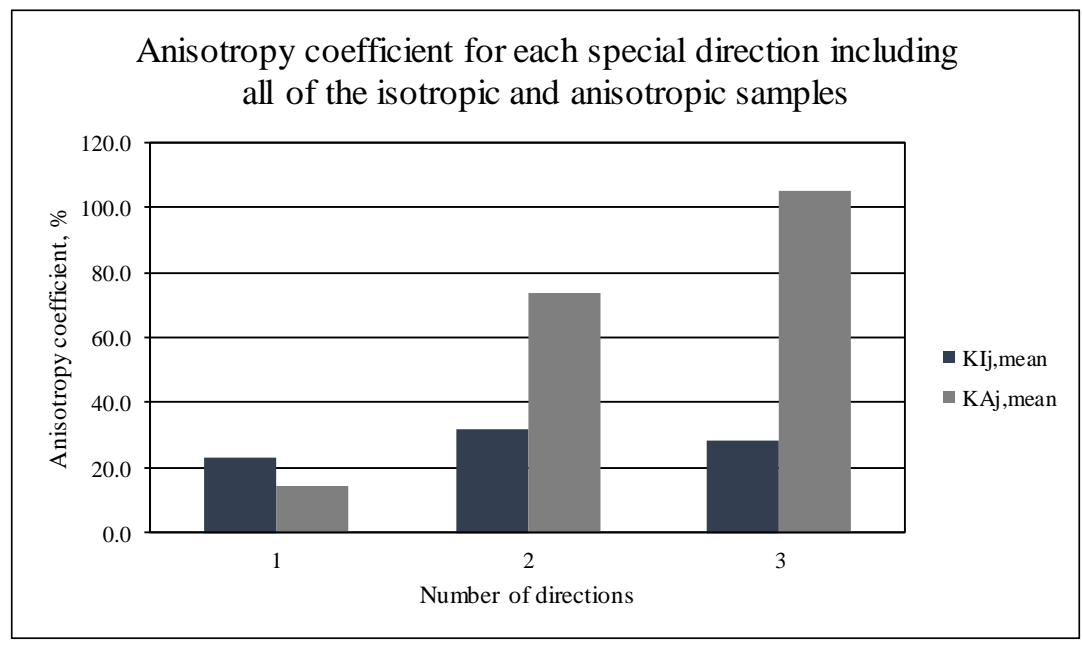

Figure 6 Anisotropy coefficients for each special direction including all of the isotropic and anisotropic samples 
Statistical analysis of the obtained results for isotropic and anisotropic samples is presented in the Table 1 and Table 2.

Table 1 Statistical analysis of the obtained results for isotropic samples

\begin{tabular}{lllllll}
\hline $\begin{array}{l}\text { Statistical } \\
\text { parameter }\end{array}$ & $D I_{i, \max } \%$ & $D I_{i, \min } \%$ & $D I_{j, \max } \%$ & $D I_{j, \min } \%$ & $K I_{i, \text { mean }} \%$ & KI $I_{j, \text { mean }} \%$ \\
\hline Min & 2.13 & 0.13 & 12.40 & 13.74 & 0.26 & 23.35 \\
Max & 8.92 & 8.38 & 15.93 & 20.25 & 15.37 & 31.71 \\
Mean & 5.49 & 3.99 & 14.17 & 16.95 & 7.55 & 27.83 \\
Range & 6.79 & 8.25 & 3.53 & 6.51 & 15.11 & 8.36 \\
StDev. & 1.76 & 2.14 & 1.76 & 3.26 & 3.92 & 4.21 \\
CV & 0.32 & 0.54 & 0.12 & 0.19 & 0.52 & 0.15 \\
\hline
\end{tabular}

Table 2 Statistical analysis of the obtained results for anisotropic samples

\begin{tabular}{lcccccc}
\hline $\begin{array}{l}\text { Statistic. } \\
\text { param. }\end{array}$ & $D A_{i, \max } \%$ & $D A_{i, \min } \%$ & $D A_{j, \max } \%$ & $D A_{j, \min } \%$ & $K A_{i, \text { mean }} \%$ & $K A_{j, \text { mean }} \%$ \\
\hline Min & 19.89 & 29.32 & 8.51 & 7.82 & 48.78 & 14.48 \\
Max & 42.08 & 229.00 & 35.90 & 114.43 & 148.29 & 105.15 \\
Mean & 30.97 & 79.55 & 23.53 & 57.30 & 97.65 & 64.47 \\
Range & 22.19 & 199.68 & 27.39 & 106.61 & 99.51 & 90.66 \\
StDev. & 3.91 & 34.71 & 13.88 & 53.72 & 18.92 & 46.04 \\
CV & 0.13 & 0.44 & 0.59 & 0.94 & 0.19 & 0.71 \\
\hline
\end{tabular}

As we can see in the Figures, review of the statistical data processing confirms the differences between investigated samples. Range implies difference between minimum and maximum. StDev, known as standard deviation, is expressed as a measure of the deviation of the mean value. $\mathrm{CV}$ - coefficient of variation is manifested as a ratio of standard deviation and mean value. These are some of the important statistical parameters which are used to investigate quantitative indicators of the anisotropy. 


\section{CONCLUSION}

The benefits of using ultrasonic in rock mass investigations are numerous and become necessary part of testing program more and more. This is especially significantly due to the fact that ultrasonic investigation is the way of testing without fracture of rock samples.

Measuring longitudinal elastic waves propagation velocities, we investigated quantitative indicators of anisotropy on the isotropic and anisotropic samples. In order to avoid measurement errors, interval numbers are applied to compute these indicators. Uncertainty of input parameters implies defining a boundaries above these data thereby increasing the flexibility and reliability of the computational process. Comparative analysis between these samples shows a highly difference of quantitative indicators of anisotropy. Isotropic samples are more uniform and more compact than anisotropic samples. The proof is that the obtained results are approximately the same for both approaches of determining the degree of anisotropy. The existence of that high similarity clearly indicates that it is an isotropic medium.

During modelling of the anisotropic rock masses, knowing of the quantitative indicators of anisotropy would be of great use for creating reliable models. Further researches can be related to determine correlation between other mechanical properties such as Young's modulus, Poisson's ratio, bulk density and compressive strength in order to create an dynamic model of anisotropy investigation.

\section{Acknowledgement}

This paper is a part of the Projects TR 36014 and TR 33029 under the auspices of the Ministry of Education, Science and Technological Development, Republic of Serbia.

\section{REFERENCES}

ALEFELD G. and MAYER G. (2000) Interval analysis: theory and applications. Journal of Computational and Applied Mathematics, 121, pp. 424-464.

ESBERT R. M. (2007) Alteration of granite stone used in building construction. Materiales de Construcción, 57, pp. 77-89.

FAVARO M. et al. (2006) Evaluation of polymers for conservation treatments of outdoor exposed stone monuments, Part I. Photo-oxidative weathering. Polymer Degradation and Stability, 91, pp. 3083-3096.

FORT R., DE BUERGO M. A. and PEREZ-MONSERRAT E. M. (2013) Nondestructive testing for the assessment of granite decay in heritage structures compared to quarry stone. International Journal of Rock Mechanics and Mining Sciences, 61, pp. 96305. 
GLIGORIĆ M., MAJSTOROVIĆ J. and LUTOVAC S. (2017) Ispitivanje anizotropije stenskih masa merenjem brzine prostiranja ultrazvučnih talasa. In: Zbornik radova sa $V$ naučno - stručnog skupa „Podzemna eksploatacija mineralnih sirovina 2017“, Beograd. Beograd: Univerzitet u Beogradu - Rudarsko - geološki fakultet.

JASIEHKO J. and STAWISKI B. (2001) Analysis of cracking plane geometry in old sandstone columns. Materials and Structures, 34, pp. 248-252.

KAHRAMAN S. (2007) The correlations between the saturated and dry P-wave velocity of rocks. Ultrasonics, 46, pp. 341-348.

KILIC O. (2006) The influence of high temperatures on limestone P-wave velocity and Schmidt hammer strength. International Journal of Rock Mechanics and Mining Sciences, 43, pp. 980-986.

MELAKU M. T. (2007) Velocity Anisotropy of shales and sandstones from core sample and well log on the Norwegian Continental shelf. Thesis (MSc), Faculty of Mathematics and Natural Sciences, University of Oslo

MOORE R. E., KEARFOTT R. B. and CLOUD M. J. (2009) Introduction to interval analysis. Philadelphia: Society for Industrial and Applied Mathematics.

MYRIN M. and MALAGA K. (2006) A case study on the evaluation of consolidation treatment of Gotland sandstone by use of ultrasound pulse velocity measurements. In: Heritage, weathering and conservation. 2nd ed. London: Taylor and Francis Group plc; pp. 749-756

PETRUŽÁLEK M. et al. (2008) Changes of anisotropy of p-wave velocity propagation during deformation process of rock samples. In: 28-European Conf. AE Testing, Ewgae 2008, Cracow UT. Cracow.

SOUSA L. M. O. et al. (2005) Influence of microfractures and porosity on the physicomechanical properties and weathering of ornamental granites. Engineering Geology, 77, pp. 153-168.

TRINDADE R. M. P. et al. (2010) An Interval Metric. In: New Advanced Technologies. InTech.

VALDELON L., DE FREITAS M.H. and KING M.S. (1996) Assessment of the quality of building stones using signals processing procedures, Quarterly Journal of Engineering Geology and Hydrogeology, 29, pp. 299-308.

VASANELLI E. et al. (2015) Ultrasonic pulse velocity for the evaluation of physical and mechanical properties of a highly porous building limestone. Ultrasonics, 60, pp. 33 -40 .

YAVUZ H. et al. (2006) Estimating the index properties of deteriorated carbonate rocks due to freeze-thaw and thermal shock weathering. International Journal of Rock Mechanics and Mining Sciences, 43, pp. 767-775. 